Article

\title{
Effectiveness of Different Rest Intervals Following Whole-Body Vibration on Vertical Jump Performance between College Athletes and Recreationally Trained Females
}

\author{
Nicole C. Dabbs ${ }^{1, *}$, Jon A. Lundahl ${ }^{2, \dagger}$ and John C. Garner ${ }^{2, \dagger}$
}

1 Biomechanics and Sport Performance Laboratory, Department of Kinesiology, California State University, San Bernardino, 5500 University Parkway, San Bernardino, CA 92407, USA

2 Applied Biomechanics Laboratory, Health, Exercise Science, and Recreation Management Department; The University of Mississippi, P.O. Box 1848, Oxford, MS 38677, USA; E-Mails: jonludndahl@gmail.com (J.A.L.); jcgarner@olemiss.edu (J.C.G.)

$\dagger$ These authors contributed equally to this work.

* Author to whom correspondence should be addressed; E-Mail: ndabbs@csusb.edu; Tel.: +1-909-537-7565; Fax: +1-909-537-7085.

Academic Editor: Lee E. Brown

Received: 4 August 2015 / Accepted: 15 September 2015 / Published: 18 September 2015

\begin{abstract}
The purpose of this study was to evaluate the effect of different rest intervals following whole-body vibration on counter-movement vertical jump performance. Sixteen females, eight recreationally trained and eight varsity athletes volunteered to participate in four testing visits separated by $24 \mathrm{~h}$. Visit one acted as a familiarization visit where subjects were introduced to the counter-movement vertical jump and whole-body vibration protocols. Visits 2-4 contained 2 randomized conditions. Whole-body vibration was administered in four bouts of $30 \mathrm{~s}$ with $30 \mathrm{~s}$ rest between bouts. During whole-body vibration subjects performed a quarter squat every $5 \mathrm{~s}$, simulating a counter-movement vertical jump. Whole-body vibration was followed by three counter-movement vertical jumps with five different rest intervals between the vibration exposure and jumping. For a control condition, subjects performed squats with no whole-body vibration. There was a significant $(p<0.05)$ main effect for time for vertical jump height, peak power output, and relative ground reaction forces, where a majority of individuals max jump from all whole-body vibration conditions was greater than the control condition. There were significant $(p<0.05)$ group differences, showing that varsity athletes had a greater vertical jump height and peak power output
\end{abstract}


compared to recreationally trained females. There were no significant $(p>0.05)$ group differences for relative ground reaction forces. Practitioners and/or strength and conditioning coaches may utilize whole-body vibration to enhance acute counter-movement vertical jump performance after identifying individuals optimal rest time in order to maximize the potentiating effects.

Keywords: rest time; counter-movement; warm-up; athletic women

\section{Introduction}

Identifying key variables in an athlete's performance is essential to improving athletic performance. It is common to use traditional training techniques, such as strength training, plyometrics, and weightlifting and it may be beneficial to incorporate non-traditional techniques to further enhance performance [1-3]. It has become increasingly popular to incorporate non-traditional training modalities such as, whole-body vibration (WBV) [4-8] to achieve performance enhancement.

Whole-body vibration uses a platform that oscillates, sending vibration to the whole body while standing on the platform. It has been shown to improve upper and lower body muscular activity in both trained and untrained individuals [7,9-16]. Previous research indicates WBV exposure at a moderate intensity is safe and effective in stimulating the neuromuscular system [4] and has been shown to induce non-voluntary muscle contractions [17]. Power output is a key variable for sports performance and previous research have shown an increase in power production by facilitation of an explosive strength effort $[9,18,19]$ leading to augmentation of performance via muscular strength and motor function [20,21]. Bouts of WBV exposure have also been seen to improve sprinting and jumping performance [5,8,10,22,23], with little or no effort by the individuals [24]. Although many studies has shown positive effects, there are several studies have shown no performance enhancements following WBV [5,7,12,25-27], resulting in inconsistency in previous literature.

Warming up prior to any physical performance is highly recommended and a widely accepted and acknowledged practice. WBV is increasingly being utilized as a warm-up for its potentiating effects prior to performance $[5,8,13,16,24,28,29]$, to prepare the body for active performance instead of traditional warm-up techniques [30-32]. Further, WBV has been used passively and/or combined with active movements due to its reported acute performance enhancing effects $[5,8]$. The acute lower body neuromuscular activation from WBV [22,33] may be beneficial in many power sports.

Numerous variables during WBV exposure can affect optimal performance outcomes such as frequency, amplitude, duration, rest intervals, platform type, and population tested [8,22,23,34]. Several combinations of these variables have been manipulated in previous research, attempting to identify optimal performance. Rest intervals, specifically, have been shown to effect performance outcomes, varying from too short of rest with possible over stimulation of the neuromuscular system or too long of rest with possible dissipating effects [8,22]. Therefore, to increase performance variables, optimal rest intervals are critical when utilizing whole-body vibration. Previous research have shown conflicting results using varying rest intervals following acute bouts of WBV, from immediately post to 
10 min $[5,8,10,12,22,23,35,36]$. To our knowledge there is no current literature that has investigated this vibration protocol with these specific rest times comparing athletes versus non-athletes.

Therefore, the purpose of this study was to investigate the effect of different rest intervals following WBV on vertical jump (VJ) performance in female recreationally trained and varsity athletes. Identifying optimal rest intervals following WBV exposure is critical to maximize vertical jump performance in trained individuals. Additionally, identifying differences between female varsity athletes and recreationally trained females will allow conclusions to be made on the applications of WBV exposure to different trained populations.

\section{Material and Methods}

\subsection{Subjects}

Sixteen females, recreationally trained ( $n=8$, age: $22 \pm 1$ year, height: $162.87 \pm 2.6 \mathrm{~cm}$, body mass: $64.35 \pm 4.64 \mathrm{~kg})$ and varsity athletes $(n=8$, age: $20 \pm 1$ year, height: $168.19 \pm 7.73 \mathrm{~cm}$, body mass: $61.35 \pm 9.68 \mathrm{~kg}$ ) volunteered to participate in four testing visits. The participants were selected randomly from responders to fliers distributed over the university campus, and by word-of mouth.

Participants who were recreationally trained were defined as individuals who within the last year participated in lower body strength and power activities about three times a week. Varsity athletes were defined as highly trained athletes currently on a Division I athletic team. Participants were asked to refrain from any physical activity $24 \mathrm{~h}$ prior to testing and were excluded if they reported any lower body orthopedic injury or musculoskeletal injury within the past year. Each visit was within plus or minus one hour from initial to all proceeding visits, separated by at least $24 \mathrm{~h}$. Participants were asked to wear comfortable clothing and the same shoes for each visit. Diet and hydration were not recorded but participants were asked to keep fluid and food consistent throughout the duration of the study. Each subject read and signed a university Institutional Review Board approved informed consent form prior to participation.

\subsection{Study Design}

The purpose of the study was to investigate acute performance potentiation following WBV exposure as it might be used in a potentiation warm-up procedure. Therefore, this study used a mixed factor-repeated measures design by having subjects perform five different rest interval conditions and comparing VJ performance to a control condition without WBV in recreationally trained and varsity athletes. Rest intervals ranged from immediate post to four minutes post.

\subsection{Procedures}

Visit one served as a familiarization session, which included completing the informed consent, anthropometric measurements, familiarization with the countermovement vertical jump (CMVJ) and WBV protocol. During the familiarization session, each subject completed three CMVJ's to assess variability; if the three CMVJ's exceeded 5\% difference in jump height, they were asked to return to the lab on a subsequent day to complete another three jumps until the criterion was met. Participants then performed six experimental conditions in three days with two conditions per day [23] separated by a 
10 min rest period. This rest period was deemed sufficient since previous literature has shown that WBV is ineffective after $10 \mathrm{~min}$ of exposure [22]. The order in which the conditions were performed was randomized and days were separated by at least $24 \mathrm{~h}$.

WBV was performed on an AIRadaptive (Power Plate, Inc.) vibration platform, which administered a tri-axial vibration frequency at $30 \mathrm{~Hz}[5,22]$ and an amplitude setting low (2-4 mm). WBV sessions entailed four bouts of $30 \mathrm{~s}$ [23] for a total of two minutes of vibration exposure with $30 \mathrm{~s}$ rest between bouts. During WBV, subjects performed quarter squats [23,37] every $5 \mathrm{~s}$ while also simulating the arm swing used in a CMJ's. Participants were instructed to step off the plate during the bouts of rest. Following WBV exposure subjects were instructed to walk quickly to the force plate ( 15 feet) to complete their rest interval for that condition followed by the three CMVJ's.

One condition served as a control during which participants stood on the vibration platform with no vibration, completed the squatting protocol then immediately performed three CMVJ's. The other five conditions used rest intervals of either immediately post, $0.5 \mathrm{~min}, 1 \mathrm{~min}, 2 \mathrm{~min}$, or $4 \mathrm{~min}$ followed by three CMVJ's [22]. In addition, each subjects max value during experimental conditions for each variable (vertical jump height, peak power output, relative ground reaction forces), regardless of rest interval, was analyzed as another condition, thereby making seven conditions overall.

During all conditions participants were instructed to begin with their arms at a 90-degree angle then perform a CMVJ to a self-selected depth with arm swing and jump as high and as explosively as possible. Fifteen seconds of rest separated each jump and all jumps were performed on an AMTI force plate (Advanced Mechanical Technology, Inc., Watertown, MA, USA). A Vertec ${ }^{\circledR}$ (Columbus, OH, USA) was used as a visual target and to measure jump height to the nearest half-inch and was positioned next to the force plate. Vertical jump height (VJH) was measured and peak power output (PPO) was calculated using the Sayers equation [38]. Peak relative ground reaction force (rGRF) was sampled at $1000 \mathrm{~Hz}$ and maximum values from the highest jump from each condition were used for analysis. After completing one condition subjects sat in a chair with no active movement for $10 \mathrm{~min}$ then completed the second condition for that visit.

\subsection{Statistical Analyses}

All statistical procedures were conducted using the Statistical Package for the Social Sciences (PASW 20.0 for Windows, SPSS, Inc., Chicago, IL, USA). An a-priori alpha was set at 0.05 to determine significance. Differences between groups for height, weight, and age were analyzed with a one-way ANOVA. Differences in VJH, Peak Power, and rGRF between conditions were analyzed with a $2 \times 7$ (training status by condition) mixed factor ANOVA.

\section{Results}

There were no significant differences between recreationally trained and athletes in height $(p=0.08)$, weight ( $p=0.44)$, and age $(p=0.07)$. There was no significant interaction for training status and time for VJH $(p=0.18)$, PPO ( $p=0.18)$, and $\operatorname{rGRF}(p=0.97)$. There was a significant main effect for time for VJH $(p<0.001)$, PPO ( $p<0.001)$, and rGRF ( $p=0.01)$, where each individuals max jump from all WBV conditions was greater than the control condition (Figure 1), however no significant $(p>0.05)$ differences were found between all other conditions (Table 1). There was a significant group differences, 
showing that varsity athletes had a greater VJH $(p<0.001)$ and PPO $(p<0.001)$ compared to recreationally trained females. There were no significant ( $p=0.96$ ) group differences for rGRF (Figure 2 ). Additionally, where each participant reached their max jump during the experimental conditions is list here: $\mathrm{IM}=4,0.5 \min =2,1 \mathrm{~min}=3,2 \mathrm{~min}=6$, and $4 \mathrm{~min}=1$.

Table 1. Vertical Jump Height (VJH), Peak Power Output (PPO), and relative ground reaction force (rGFR) maximum values and values for each condition (mean \pm SD) for varsity athletes and recreationally trained.

\begin{tabular}{|c|c|c|c|c|c|c|}
\hline \multirow{2}{*}{ Conditions } & \multicolumn{2}{|c|}{ VJH (cm) } & \multicolumn{2}{|c|}{ PPO (W) } & \multicolumn{2}{|c|}{ rGRF (N) } \\
\hline & Varsity & Rec & Varsity & Rec & Varsity & Rec \\
\hline Control & $50.48 \pm 6.78$ & $42.06 \pm 5.74 *$ & $4062.50 \pm 411.54$ & $3551.79 \pm 348.27 *$ & $1608.91 \pm 510.74$ & $1613.90 \pm 356.66$ \\
\hline $0 \mathrm{~min}$ & $50.95 \pm 7.0$ & $41.91 \pm 4.55 *$ & $4091.41 \pm 425.11$ & $3542.15 \pm 276.42 *$ & $1616.72 \pm 502.40$ & $1599.02 \pm 363.28$ \\
\hline $5 \min$ & $51.91 \pm 6.84$ & $39.84 \pm 5.72 *$ & $4149.23 \pm 415.01$ & $3416.88 \pm 347.05 *$ & $1629.38 \pm 561.86$ & $1645.71 \pm 327.41$ \\
\hline $1 \min$ & $48.89 \pm 9.77$ & $40.95 \pm 6.29 *$ & $3966.14 \pm 592.85$ & $3484.34 \pm 381.57 *$ & $1626.78 \pm 468.27$ & $1628.41 \pm 289.14$ \\
\hline $2 \min$ & $50.16 \pm 6.18$ & $43.33 \pm 2.29 *$ & $4043.23 \pm 375.40$ & $3628.88 \pm 145.32 *$ & $1629.38 \pm 525.42$ & $1600.58 \pm 384.68$ \\
\hline $4 \min$ & $50.80 \pm 8.56$ & $40.64 \pm 6.40 *$ & $4081.78 \pm 519.59$ & $3465.06 \pm 388.73 *$ & $1662.43 \pm 634.03$ & $1643.70 \pm 387.99$ \\
\hline Max & $53.34 \pm 7.25 \#$ & $44.45 \pm 3.84 * \#$ & $4235.95 \pm 439.96 \#$ & $3696.33 \pm 233.10 * \#$ & $1727.36 \pm 387.99 \#$ & $1692.04 \pm 355.47 \#$ \\
\hline
\end{tabular}

* indicates significant $(p<0.05)$ differences between varsity and recreational athletes; \# indicates significant $(p<0.05)$ differences greater than control.

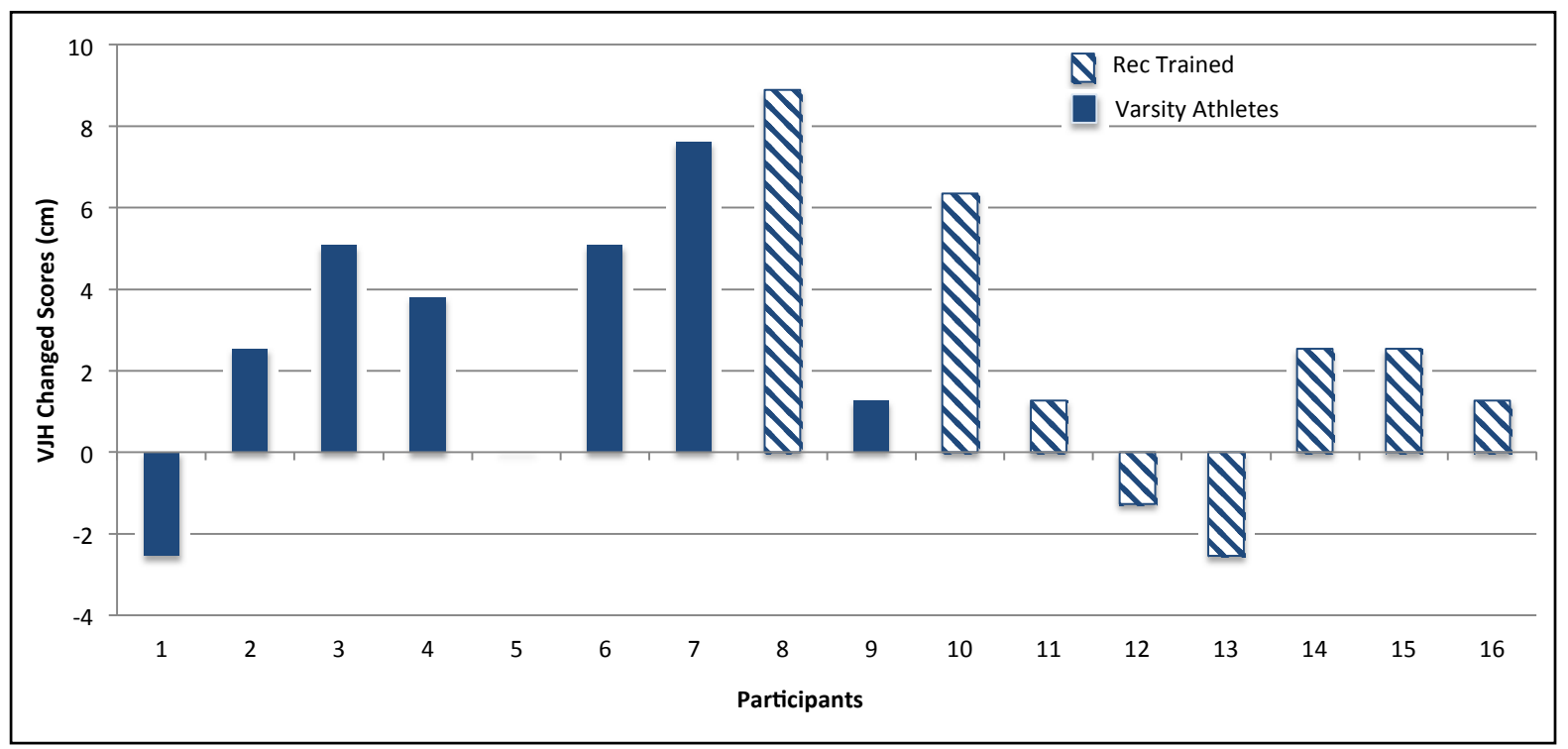

Figure 1. Individual differences between control and maximal VJ height (cm). 


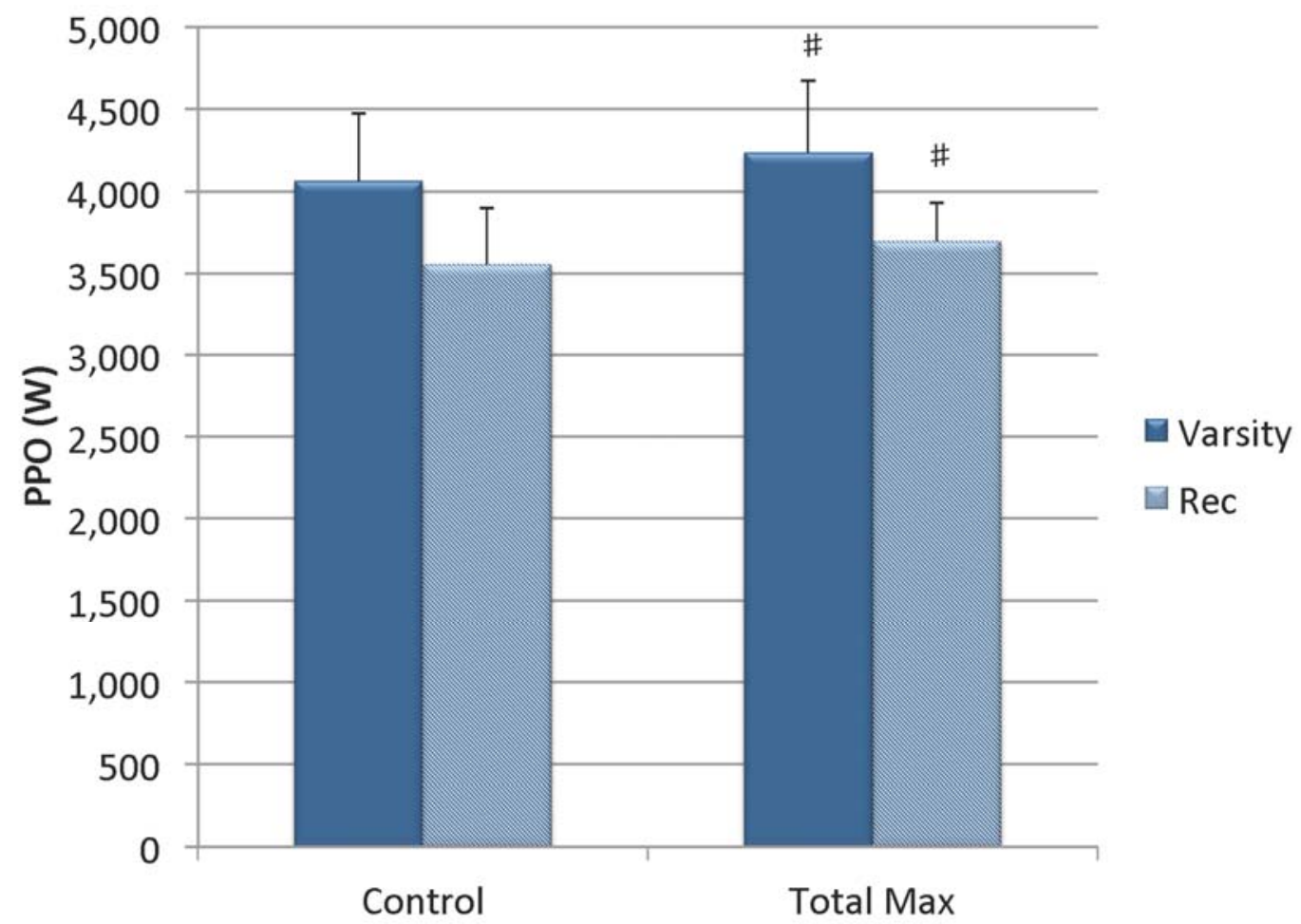

Figure 2. Differences between control condition and max condition for Peak Power Output (PPO) for varsity and recreationally trained females. \# indicates significant $(p<0.05)$ differences between conditions; * indicates significant $(p<0.05)$ differences between groups.

\section{Discussion}

The aim of the current investigation was to determine the optimal rest interval following bouts of WBV on vertical jump performance variables. The main findings were that following WBV; VJH, PPO, and rGRF showed individual increases during one of the rest interval conditions with WBV exposure compared to the control condition (no WBV). Indicating that individuals respond differently to the WBV exposure, resulting in individual optimal rest times following WBV exposure. Another finding in this investigation was that for all conditions varsity female athletes had a greater VJH and PPO compared to the recreationally trained females.

In a previous study, the researchers found individual optimal rest intervals between WBV exposure and vertical jumping performance, similar to the current investigation using similar protocols but comparing recreationally trained males and females only [8] instead of an athletic population. These similarities indicate that regardless of training status or sex, a majority of individuals responded to the WBV exposure and had an increased vertical jump performance. The only other methodical difference from this particular previous research was that the current study used a tri-axial WBV platform, while the previous study used a pivotal vibration platform. Therefore, similar results in both studies indicate that the type of vibration platform exposure has similar benefits on vertical jump performance as well. This is a positive finding, since most research studies using WBV are done on a variety of platforms, it is often uncertain if researchers can make comparisons. Previous studies have examined the differences using different types of WBV platforms [34,39], since different platforms have different oscillatory motions. A recent study examined the differences in three platform types and found no differences on 
their acute vertical jump performance [34]. The researcher concluded that different devices could be used similarly for acute vertical jump performance bouts.

Other key variables are critical when using WBV for performance enhancement such as frequency, amplitude, rest times, and vibration exposure time. Frequency can easily be changed on most devices and can be described as the intensity of the vibration where amplitude can be described as the volume. Previous research has shown that 1 min rest post vibration exposure and a frequency of $30 \mathrm{~Hz}$ were optimal for vertical jump performance increases [22,23]. The frequency exposure was similar to the current study, however our study did differ in the time exposure of WBV. Bedient and Adams [22,23] exposed participants to one bout of $30 \mathrm{~s}$ of WBV and found an increase in CMVJ, resulting in a homogenous optimal rest interval of 1 min post when compared to immediate post, $5 \mathrm{~min}$, or $10 \mathrm{~min}$. In contrast, our study exposed subjects to four bouts of $30 \mathrm{~s}$ of vibration with a 1:1 rest ratio, and found individual optimal rest intervals for an increase in vertical jump performance. In contrast, another study examined varied frequencies and durations of vibration exposures and found no differences in vertical jump performance, indicating no conclusive frequency or vibration exposure time [34].

There are some research studies that conclude that using WBV prior to vertical jump performance induces a post-activation potentiation (PAP) response [5,35]. PAP has been shown to increase voluntary force development during muscle actions [40] but can induce fatigue if the intensity is too high or there is not sufficient rest periods before performance [41]. PAP has been shown to vary on an individual basis [42], some individuals have different optimal rest times and fatigue effects, which should be considered when inducing PAP [41]. Previous research that uses WBV for PAP to enhance vertical jump performance have seen positive effects at enhancing acute performance [5,8,35]. In the current investigation, no differences between rest intervals were found, however when taking individual max vertical jump values with WBV and comparing it to the control group, individuals showed an increase in vertical jump performance. Thus, the increases in vertical jump performance induced by WBV may have been sufficient enough to induce PAP but limited enough not to induce fatigue.

The current study compared female college athletes to recreationally trained females. As we expected, the college athletes performed at a higher level with greater vertical jump performance compared to the recreationally trained individuals. These results are most likely contributed to college athletes having a higher level of training, increasing their potential to develop a higher force generating capacity [42], resulting in a higher VJH. Additionally, the athletic population may have more experience with vertical jumping allowing them to have greater vertical jumping performance accompanied with a higher level of strength and conditioning periodized programs required by their college sport. However, there have been conflicting results in a variety of studies with athletic and recreationally trained individuals with WBV and jumping performance $[7,8,43,44]$. In the current study, there were no group differences in the rGRF but there was a difference for VJH and PPO. This may be due to the use of the Vertec ${ }^{\circledR}$ device to measure VJH instead of estimating flight time from the force plate. The use of the Vertec ${ }^{\circledR}$ doesn't account for changes in arm reach or trunk twist during the jumps, which may modify scores [45]. On the other hand, VJH estimation could be altered with landing mechanics when using the force plate [45].

Our study has a few potential limitations that may have influenced the results of our study. One may have been our testing protocol, there were two conditions performed in one testing day with a 10 min washout period between conditions. This was randomized to control for any learning effects however, it is possible that one condition influenced the other. Research suggests that after $10 \mathrm{~min}$, WBV has no 
effect on vertical jump performance [22], therefore we do not suspect a learning effect occurred. Another limitation may have been that since our data reflects the maximum $\mathrm{VJH}$ for each condition, it is possible that it may have affected subsequent VJ performance. However, previous research shows that when analyzing kinetics during VJ performance there were no subsequent effects shown [46]. It is important to note that three of the participants actually perform better in the control condition compared to the experimental condition. This has been shown in other studies and has been suggested that there are responder and non-responders to WBV treatment [8]. Another important limitation to mention is the small power due to the sample size. A critical factor is the type of WBV platform utilized. The present study utilized a tri-axial platform where others [8,22,23,35] have utilized similar and other types (e.g., vertical, sliding horizontal, and pivotal), which may affect the outcome of the study.

\section{Conclusions}

Post activation potentiation was induced using whole-body vibration exposure on vertical jump performance on eight female college athletes and eight recreationally trained females. Four sets of $30 \mathrm{~s}$ bouts of WBV prior to vertical jump performance showed statistically significant differences between individual maximum jumps at different rest times with WBV compared to the control (no WBV). Therefore, it is recommended that practitioners determine individual optimal rest times following WBV prior to high level performance to obtain greatest potentiating effects. Acquiring individual optimal rest times prior to performance would allow individuals to maximum their potentiating acute effects in a single explosive movement. Therefore, this study specifically applies to female athletes that perform single bouts of explosive movements such as, high jumpers and long jumpers. Once optimal rest time is determined, it will allow for optimal performance during competition. This investigation has shown these effects to be applied to female athletic and recreationally trained individuals.

\section{Author Contributions}

Nicole Dabbs, Jon Lundahl, and John Garner were involved in study design, data collection, data interpretation, and manuscript writing.

\section{Conflicts of Interest}

The authors declare no conflict of interest.

\section{References}

1. Kraemer, W.J.; Ratamess, N.A. Fundamentals of resistance training: Progression and exercise prescription. Med. Sci. Sports Exerc. 2004, 36, 674-688.

2. Newton, R.U.; Gerber, A.; Nimphius, S.; Shim, J.K.; Doan, B.K.; Robertson, M.; Pearson, D.R.; Craig, B.W.; Häkkinen, K.; Kraemer, W.J. Determination of functional strength imbalance of the lower extremities. J. Strength Cond. Res. 2006, 20, 971-977.

3. Newton, R.U.; Kraemer, W.J.; Hakkinen, K. Effects of ballistic training on preseason preparation of elite volleyball players. Med. Sci. Sports Exerc. 1999, 31, 323-330. 
4. Cardinale, M.; Bosco, C. The use of vibration as an exercise intervention. Exerc. Sport Sci. Rev. 2003, 31, 3-7.

5. Cormie, P.; Deane, R.S.; Triplett, N.T.; McBride, J.M. Acute effects of whole-body vibration on muscle activity, strength, and power. J. Strength Cond. Res. 2006, 20, 257-261.

6. Da Silva, M.E.; Fernandez, J.M.; Castillo, E.; Nuñez, V.M.; Vaamonde, D.M.; Poblador, M.S.; Lancho, J.L. Influence of vibration training on energy expenditure in active men. J. Strength Cond. Res. 2007, 21, 470-475.

7. Dabbs, N.C.; Brown, L.E.; Coburn, J.W.; Lynn, S.K.; Tran, T.T.; Biagini, M.S. Effect of Whole-body vibration warm-up on bat speed in women softball players. J. Strength Cond. Res. 2010, 24, 2296- 2299.

8. Dabbs, N.C.; Muñoz, C.X.; Tran, T.T.; Brown, L.E.; Bottaro, M. Effect of different rest intervals after whole-body vibration on vertical jump performance. J. Strength Cond. Res. 2011, 25, 662-667.

9. Bosco, C.; Cardinale, M.; Tsarpela, O. Influence of vibration on mechanical power and electromyogram activity in human arm flexor muscles. Eur. J. Appl. Physiol. Occup. Physiol. 1999, 79, 306-311

10. Bullock, N.; Martin, D.T.; Ross, A.; Rosemond, C.D.; Jordan, M.J.; Marino, F.E. Acute effect of whole-body vibration on sprint and jumping performance in elite skeleton athletes. J. Strength Cond. Res. 2008, 22, 1371-1374.

11. Cochrane, D.J.; Hawke, E.J. Effects of acute upper-body vibration on strength and power variables in climbers. J. Strength Cond. Res. 2007, 21, 527-531.

12. Cochrane, D.J.; Stannard, S.R. Acute whole body vibration training increases vertical jump and flexibility performance in elite female field hockey players. Br. J. Sports Med. 2005, 39, 860-865.

13. Dallas, G.; Kirialanis, P.; Mellos, V. The acute effect of whole body vibration training on flexibility and explosive strength of young gymnasts. Biol. Sport 2014, 31, 233-237.

14. Di Loreto, C.; Ranchelli, A.; Lucidi, P.; Murdolo, G.; Parlanti, N.; de Cicco, A.; Tsarpela, O.; Annino, G.; Bosco, C.; Santeusanio, F.; et al. Effects of whole-body vibration exercise on the endocrine system of healthy men. J. Endocrinol. Investig. 2004, 27, 323-327.

15. Jacobs, P.L.; Burns, P. Acute enhancement of lower-extremity dynamic strength and flexibility with whole-body vibration. J. Strength Cond. Res. 2009, 23, 51-57.

16. Despina, T.; George, D.; George, T.; Sotiris, P.; Alessandra, D.C.; George, K.; Maria, R.; Stavros, K. Short-term effects of whole-body vibration training on balance, flexibility and lower limb explosive strength in elite rhythmic gymnasts. Hum. Mov. Sci. 2014, 33, 149-158.

17. Issurin, V.B. Vibrationss and their applications in sport: A review. J. Sports Med. Phys. Fit. 2005, 45, 424-436.

18. Issurin, V.B.; Tenenbaum, G. Acute and residual effects of vibratory stimulation on explosive strength in elite and amateur athletes. J. Sports Sci. 1999, 17, 177-182.

19. Poston, B.; Holcomb, W.R.; Guadagnoli, M.A.; Linn, L.L. The acute effects of mechanical vibration on power output in the bench press. J. Strength Cond. Res. 2007, 21, 199-203.

20. Bosco, C.; Cardinale, M.; Tsarpela, O.; Colli, R.; Tihanyi, J.; von Duvillard, S.P.; Viru, A. The influence of whole body vibration on jumping performance. Biol. Sports. 1998, 15, 157-614.

21. Bosco, C.; Colli, R.; Introini, E.; Cardinale, M.; Tsarpela, O.; Madella, A.; Tihanyi, J.; Viru, A. Adaptive responses of human skeletal muscle to vibration exposure. Clin. Physiol. 1999, 19, 183-187. 
22. Adams, J.B.; Edwards, D.; Serravite, D.H.; Bedient, A.M.; Huntsman, E.; Jacobs, K.A.; del Rossi, G.; Roos, B.A.; Signorile, J.F. Optimal frequency, displacement, duration, and recovery patterns to maximize power output following acute whole-body vibration. J. Strength Cond. Res. 2009, 23, 237-245.

23. Bedient, A.M.; Adams, J.B.; Edwards, D.A.; Serravite, D.H.; Huntsman, E.; Mow, S.E.; Roos, B.A.; Signorile, J.F. Displacement and frequency for maximizing power output resulting from a bout of whole-body vibration. J. Strength Cond. Res. 2009, 23, 1683-1687.

24. Rittweger, J.; Beller, G.; Felsenberg, D. Acute physiological effects of exhaustive whole-body vibration exercise in man. Clin Physiol. 2000, 20, 134-142.

25. De Ruiter, C.J.; van der Linden, R.M.; van der Zijden, M.J.; Hollander, A.P.; de Haan, A. Short-term effects of whole-body vibration on maximal voluntary isometric knee extensor force and rate of force rise. Eur. J. Appl. Physiol. 2003, 88, 472-475.

26. Kelly, S.B.; Alvar, B.A.; Black, L.E.; Dodd, D.J.; Carothers, K.F.; Brown, L.E. The effect of warm-up with whole-body vibration vs. cycle ergometry on isokinetic dynamometry. J. Strength Cond. Res. 2010, 24, 3140-3143.

27. Menefee, K.; Switzler, C.; Podlog, L.; Hickes-Little, C. The acute effects of two different foot positions during whole body vibration on vertical jump performance in females. J. Athl. Med. 2014, 2, 66-74.

28. Cochrane, D.J.; Stannard, S.R.; Sargeant, A.J.; Rittweger, J. The rate of muscle temperature increase during acute whole-body vibration exercise. Eur. J. Appl. Physiol. 2008, 103, 441-448.

29. Dallas, G.; Kirialanis, P. The effects of two different conditions of whole-body vibration on flexibility and jumping performance on artistic gymnasts. Sci. Gymnast. J. 2013, 5, 41-52.

30. Burkett, L.N.; Phillips, W.T.; Ziuraitis, J. The best warm-up for the vertical jump in college-age athletic men. J. Strength Cond. Res. 2005, 19, 673-676.

31. Chaouachi, A.; Castagna, C.; Chtara, M.; Brughelli, M.; Turki, O.; Galy, O.; Chamari, K.; Behm, D.G. Effect of warm-ups involving static or dynamic stretching on agility, sprinting, and jumping performance in trained individuals. J. Strength Cond. Res. 2010, 24, 2001-2011.

32. Gourgoulis, V.; Aggeloussis, N.; Kasimatis, P.; Mavromatis, G.; Garas, A. Effect of a submaximal half-squats warm-up program on vertical jumping ability. J. Strength Cond. Res. 2003, 17, 342-344.

33. Abercromby, A.F.; Amonette, W.E.; Layne, C.S.; McFarlin, B.K.; Hinman, M.R.; Paloski, W.H. Variation in neuromuscular responses during acute whole-body vibration exercise. Med. Sci. Sports Exerc. 2007, 39, 1642-1650.

34. Bagheri, J.; van den Berg-Emons, R.J.; Pel, J.J.; Horemans, H.L.; Stam, H.J. Acute effects of whole-body vibration on jump force and jump rate of force development: A comparative study of different devices. J. Strength Cond. Res. 2012, 26, 691-696.

35. McBride, J.M.; Nuzzo, J.L.; Dayne, A.M.; Israetel, M.A.; Nieman, D.C.; Triplett, N.T. Effect of an acute bout of whole body vibration exercise on muscle force output and motor neuron excitability. J. Strength Cond. Res. 2010, 24, 184-189.

36. Ronnestad, B.R. Acute effects of various whole-body vibration frequencies on lower-body power in trained and untrained subjects. J. Strength Cond. Res. 2009, 23, 1309-1315.

37. Cochrane, D.J.; Legg, S.J.; Hooker, M.J. The short-term effects of whole-body vibration training on vertical jump, sprint, and agility performance. J. Strength Cond. Res. 2004, 18, 828-832. 
38. Sayers, S.P.; Harackiewicz, D.V.; Harman, E.A.; Frykman, P.N.; Rosenstein, M.T. Cross-validation of three jump power equations. Med. Sci. Sports Exerc. 1999, 31, 572-577.

39. Pel, J.J.; Bagheri, J.; van Dam, L.M.; van den Berg-Emons, H.J.; Horemans, H.L.; Stam, H.J.; van der Steen, J. Platform accelerations of three different whole-body vibration devices and the transmission of vertical vibrations to the lower limbs. Med. Eng. Phys. 2009, 31, 937-944.

40. Tillin, N.; Bishop, D. Factors modulating post-activation potentiation and its effect on performance of subsequent explosive ativities. Sports Med. 2009, 39, 147-166.

41. McCann, M.R.; Flanagan, S.P. The effects of exercise selection and rest interval on postactivation potentiation of vertical jump performance. J. Strength Cond. Res. 2010, 24, 1285-1291.

42. Chiu, L.Z.; Fry, A.C.; Weiss, L.W.; Schilling, B.K.; Brown, L.E.; Smith, S.L. Postactivation potentiation response in athletic and recreationally trained individuals. J. Strength Cond. Res. 2003, 17, 671-677.

43. Burns, J.D.; Miller, P.C.; Hall, E.E. Acute Effects of Whole Body Vibration on Functional Capabilities of Skeletal Muscle; Federación Española de Asociaciones de Docentes de Educación Física: Elon, NC, USA, 2015; pp. 180-183.

44. Naclerio, F.; Faigenbaum, A.D.; Larumbe-Zabala, E.; Ratamess, N.A.; Kang, J.; Friedman, P.; Ross, R.E. Effectiveness of different postactivation potentiation protocols with and without whole body vibration on jumping performance in college athletes. J. Strength Cond. Res. 2014, 28, 232-239.

45. Ferreira, L.C.; Schilling, B.K.; Weiss, L.W.; Fry, A.C.; Chiu, L.Z. Reach height and jump displacement: Implications for standardization of reach determination. J. Strength Cond. Res. 2010, 24, 1596-1601.

46. Jensen, R.L.; Ebben, W.P. Kinetic analysis of complex training rest interval effect on vertical jump performance. J. Strength Cond. Res. 2003, 17, 345-349.

(C) 2015 by the authors; licensee MDPI, Basel, Switzerland. This article is an open access article distributed under the terms and conditions of the Creative Commons Attribution license (http://creativecommons.org/licenses/by/4.0/). 